\title{
Nicoletta Marini-Maio and Colleen Ryan-Scheutz (eds) (2008). Set the Stage! Teaching Italian through Theater.
}

\author{
Kelly C. Kingsbury
}

Set the Stage! Teaching Italian through Theater: Theories, Methods, and Practices (Yale, 2008), edited by Nicoletta Marini-Maio and Colleen Ryan-Scheutz, is a tremendously valuable contribution to the growing body of literature on drama and theatre in foreign/second language (L2) education. This volume contains eleven chapters addressing a diverse array of topics, a comprehensive director's handbook, and reflective contributions by Dario Fo, Franca Rame and Dacia Maraini, three of Italy's most prominent theatre practitioners engaged with pedagogical questions related to the learning of L2 Italian through theatre. The chapters of Set the Stage! encompass both theoretical and practical orientations toward questions of culture, theatre history, curriculum, and assessment in language learning, and they offer an array of perspectives that illuminate a variety of possible models for incorporating diverse forms of theatre within the L2 curriculum.

The first section of Set the Stage! includes an overview of theatre's place within Italian literature and culture. Following Pietro Frassica's overview of the Italian theatrical canon, William Van Watson offers an insightful commentary on the inherent theatricality of Italian cultural interactions, the possibilities this raises for cultural misunderstandings, and the concomitant potential it holds for theatrically teachable moments.

Section II focuses on the place of theatre courses in the Italian curriculum. Salvatore Banchieri, Francesca Savoia, Laura Colangelo and Colleen-Ryan Scheutz present descriptions of course structures within diverse contexts and aimed at various goals. Banchieri describes the implementation of a course blending academic content and theatrical performance within two different university settings, highlighting the modifications by which the course can be adapted to suit the particulars of each. Savoia explores the use of theatre as a bridge to assist learners in negotiating the breach between language and literature courses, emphasizing theatre's potential to improve retention across this persistent curricular divide. Colangelo and Ryan-Scheutz address the possibilities as well as the special challenges that exist for those who wish to integrate theatre at the secondary level.

The third section of the volume focuses on genres, themes and techniques specific to Italian: commedia dell'arte, opera, and the politically-engaged comedies of Dario Fo and Franca Rame. These topics are analyzed as subjects 
of content-based L2 study by Gian Giacomo Colli, Daniela Noé-Le Sassier and Frances Boyd, and Walter Valeri, who offer additional variations on the possible classroom models introduced in previous chapters.

In the fourth section, the authors approach their proposed pedagogical models from a standpoint of methods and assessment. Nicoletta Marini-Maio examines the language-literature breach earlier described by Savoia, observing that

[t] he performative approach to theater may claim a primary position in that delicate stage of the Italian curriculum in which students initiate the exploration of sophisticated literary texts while still needing to enhance their communicative competencies and develop literacy skills. In the field of Italian pedagogy it may indeed provide an invaluable contribution by filling an empty space, a space of cultural transformation, linguistic, and psychological growth. (261)

Del Fattore-Olson describes the integration of performative, linguistic, literary, historical and sociocultural elements in the L2 classroom, while Ryan-Scheutz contributes a trenchant analysis of approaches to assessment that maintain consistency with the course's communicative goals as well as satisfying institutional requirements for demonstrating proficiency and academic rigor.

Seven of the volume's contributors provide additional practical guidelines and suggestions in Section V, the Director's Handbook. These range from advice on text selection, finances, publicity, community engagement, developing English translations for audience members, to acting exercises and recommendations for beginning directors engaged in play production. An appendix includes sample course syllabi, audition documents, scene breakdowns for scheduling, warm-up activities, and an outline of expectations and duties for non-acting participants on the design team. A collection of illustrations and photographs and an afterword consisting of an interview with Dario Fo and Franca Rame, together with the prologue by Dacia Maraini, round out the volume's contents.

The special strengths of Set the Stage!'s contribution to the literature on theatre in L2 learning include the diversity of its topics and the high quality of its theoretical grounding. As Marini-Maio notes in the introduction to her chapter, practitioners of L2 theatre as a pedagogical approach generally possess enthusiasm and passion in abundance and tend to share intellectual curiosity as well as eclectic backgrounds encompassing language and literature study as well as practical theatre experience, but they

generally are reluctant to give an authoritative voice to their innovative pedagogical practices ... [b]ecause of the scarcity of theater-specific theoretical contributions in the field of L2 acquisition, they have had to develop their own teaching methods more from hands-on experience in the classroom and on the stage than from an articulated system of thought. (239)

Set the Stage! does much to elaborate the articulated system of thought the field is calling for. Likewise, Ryan-Scheutz' thorough and considered chapter 
on assessment addresses a central, lamentably undertheorized area of L2 theatre praxis. Although testing (especially in its most traditional guise) is understandably not at the forefront of instructors' concerns while they are swept up in the exigencies of producing a show, if L2 theatre is to occupy the central place in the curriculum that we would like it to, instructors must be able to demonstrate that these courses satisfy overarching institutional requirements, including meeting the demand for consistent, reliable and justifiable assessment measures. Set the Stage! offers valuable insights for moving that conversation forward. A further strength of this text is the diversity of course models described in its chapters. The contributors offer theoretical support as well as helpful descriptions for incorporating theatre as a component of a reading-centered course encompassing multiple selections and for designing a course exclusively dedicated to the performance of a single text. Alternative performance options such as scene recitals and one-act plays offer possibilities for practitioners interested in incorporating theatre in their pedagogy but intimidated by the prospect of attempting a full-scale production. The Director's Handbook in Section V is a wise inclusion for encouraging the expansion of L2 theatre in departments where it does not currently have a place in the curriculum.

Although its focus is explicitly on the teaching of Italian and the performance of Italian-language works, Set the Stage! is a useful addition to the library of theatre pedagogues across the language spectrum. The chapters addressing Italian-specific topics such as commedia dell'arte, opera, Italy's cultural theatricality and regional dialects are rounded out by discussions of issues relevant to all languages, notably the need for theatre to serve as a bridge between language acquisition and literary analysis and the importance of valid assessment in theatre performance courses. The one area that could have been augmented in Set the Stage! is the explicit analysis of how grammatical structures can be taught in context using theatrical texts and productively acquired by learners, an issue which is briefly touched on in a number of chapters. Given the need for strong persuasive arguments to justify theatre projects and courses in an era of shrinking budgets, such a topic merits at least a chapter, if not a section, of its own. Nonetheless, Marini-Maio and Ryan-Scheutz' volume represents a rich addition to the theoretical literature on theatre in L2 pedagogy that will appeal to readers well beyond the population of Italian instructors who constitute its primary audience.

\section{Bibliography:}

Marini-Maio, Nicoletta \& Ryan-Scheutz, Colleen (eds.) (2008): Set the Stage! Teaching Italian through Theater: Theories, Methods, and Practices. New Haven: Yale. 